\title{
Global Perspective on Understanding the Potential Benefits and Risks of E-Cigarette use on Head and Neck Cancer
}

\author{
Joshua E Muscat ${ }^{*}$ and Wenxue Lin \\ Department of Public Health Sciences, The Pennsylvania State University College of Medicine USA
}

\section{Introduction}

Head and neck cancer (HNC) is the seventh most common cancer worldwide [1], with 887,659 new cases in 2018 [2]. The time trends in HNC rates from 1983 to 2002 vary by geography [3]. The 2018 age-standardized rate of oral cavity varies from 1.2 per 100,000 in Western Africa to 12.9 in Southeast Asia [2]. The major risk factors are tobacco and alcohol for all HNC subsites, except for HPV infection which is likely the major cause of oropharyngeal cancer in developed countries.

The rapid emergence in the use of electronic cigarettes (e-cigarettes) could dramatically affect the risk for the development of oral cancer. Among middle and high school students in the United States (U.S.), the prevalence of e-cigarette use was $78 \%$ in 2018 [4]. In a survey of 45,971 U.S. adults and youth, a flavored e-cigarette was their first tobacco product [5]. Many who first used an e-cigarette for the first time continued to smoke them at least monthly [6].

Electronic cigarettes do not contain carcinogens present in combustible tobacco, and are considered substantially less toxic and less carcinogenic. While research has increased to understand the potential adverse health effects of e-cigarettes, its effects on oral cancer are unknown. There has been no specific research agenda established to systematically understand how e-cigarette use may affect oral cancer, and make recommendations about how such risks may be mitigated. Previous reviews have summarized the literature on e-cigarettes and non-malignant oral disease [7]. In the current paper, we discuss what is known about the possible effects of e-cigarettes on oral cancer and lay out future research goals.

\section{Epidemiology of e-cigarettes and Oral Cancer}

A starting point for understanding e-cigarettes and oral cancer would be the epidemiology of e-cigarette use and oral cancer. Because e-cigarettes have been used primarily in young individuals and for relatively short durations, case-control and cohort data are unavailable for informing on the risk. Such data may become available in the future. If e-cigarettes become a preferred method for smoking cessation, it would be feasible to study the risk of oral cancer in smokers who switched to e-cigarettes compared to smokers who continue to use combustibles. It may take decades of work to understand if there is a risk-reduction, are there dose-response effects, do effects vary by e-cigarette characteristics, flavors and population subgroups. It will be necessary to determine the moderating and mediating effects of confounders like combustible cigarettes. Alternatively, cohort studies in middle aged smokers might elucidate the effects of e-cigarettes on oral cancer. Such studies will likely require multiple exposure assessments over the years in anticipation of changing smoking/vaping habits and changes in types of products. Due to the large expense in conducting epidemiologic studies, the short duration of exposure to e-cigarette vapor among its users, and that most users are relatively young, it may be years before epidemiologic data are available to assess the risks of oral cancer.

In developing countries, the epidemiology of e-cigarettes and oral cancer might be expected to be quite different. The use of oral tobacco is endemic in many parts of Southeast Asia and Africa. The types of oral tobacco used also varies by region as well as how it is used. One common form is betel quid, which causes a much higher risk than chewing tobacco used in developed countries. Sometimes oral tobacco is used in conjunction with cigarettes. Further, the types of cigarettes used in developing countries also differ than in developed countries. Bidis are cigarettes of hand-wrapped tobacco in plant leaf and are commonly used in parts of South and Southeast Asia. They are often fruit-flavored. Bidis cause OC [8], HC and LC [9]. Bidis are generally unfiltered and therefore may have a greater carcinogenic potential than filtered cigarettes. Similar to the consideration of studies of e-cigarettes and oral cancer in developed countries, it may be decades before epidemiologic data are available to assess the risks of oral cancer in developing countries. Such studies will need to account for the various forms of oral tobacco used. The

*Corresponding author: Joshua E Muscat PhD, Department of Public Health Sciences, The Pennsylvania State University College of Medicine, MC CH69, 500 University Drive, P.O. Box 850, Hershey, PA, 17033, USA. E-mail: jmuscat@pennstatehealth.psu.edu

Accepted: November 10, 2020

Published online: November 12, 2020

Citation: Muscat JE, Lin W (2020) Global Perspective on Understanding the Potential Benefits and Risks of E-Cigarette use on Head and Neck Cancer. J Oral Cancer Res 3(1):28-30 
conduct of such studies will depend on whether future use of e-cigarettes becomes common in these countries. For example, e-cigarettes containing nicotine have been banned in Japan since 2010. E-cigarettes without nicotine are available, and are less toxic than cigarettes and possible nicotine-containing e-cigarettes.

Future ecologic studies might be able to assess the effects of nicotine-free e-cigarettes on oral cancer compared to e-cigarettes with nicotine. More critically, smoking rates continue to be very high in Japanese men. Like in Western countries, it may be possible to conduct simple epidemiologic studies using birth cohorts or other measures on the rates of oral cancer. The best methods though, as well as the most important public health question is to assess whether e-cigarettes that are used to facilitate smoking reduction or cessation reduces the subsequent risk of oral cancer. Since e-cigarettes contain numerous oxidants that have promotional effects on cancer, at levels not much lower than cigarettes [10], switching from cigarettes to e-cigarettes may have less of a benefit on oral cancer risk than is currently realized. Epidemiologic studies and animal model systems will be needed to determine whether e-cigarettes reduce oral cancer risk after cessation of tobacco smoke exposure.

\section{Effects of e-cigarettes on Human Oral Micro- biome}

Studies of the oral microbiome on oral health have been limited to the most part on their potential effect on periodontal disease, dental carries and mouth effects, which itself are possible but un-established risk factor for oral cancer, and possibly mediated by the effects from cigarette smoking. There are few studies on whether e-cigarettes are risk factors for periodontal diseases and dental caries. In one study, of 119 participants, the abundance of bacteria Porphyromonas and Veillonella was higher in e-cigarette users compared with conventional cigarette smokers or never smokers [11]. Another study found an increase in gingival inflammation in smokers switching to e-cigarettes although cytokine analysis indicated the findings were inconclusive [12]. In contrast, a larger study found higher levels of oral inflammation in cigarette-smokers compared with never-smokers but no differences between e-cigarette users and never-smokers [13].

\section{In-vitro studies}

In the absence of human data, in-vitro studies offer the potential to inform on the effects of e-cigarettes. To date, no studies have examined carcinogenic endpoints or processes in transformed oral cells. The OECM-1 human oral cavity squamous cell carcinoma cell line is an established model for squamous cell carcinoma and is characterized by a mutation in the p53 tumor suppressor [14]. Whether this cell line is suitable for studying the toxicity of e-cigarette vapor will need to be determined. In the absence of carcinogenic models, toxicity studies might be helpful to study the effects of e-cigarettes. There have been few toxicity studies to date. Exposure to e-cigarette vapor induced morphological changes in ginigiva cells, and increased L-lactate dehydrogenase activity and apoptosis [15]. Another study found that different e-cigarette flavorings had differential effects on gingiva inflammatory response [16].

These studies suggest possible effects on peridontal disease rather than oral cancer per se. Periodontitis is a possible risk factor for oral cancer but this has not been fully established $[17,18]$. One study examined the in vitro toxicity in oral cells from a third-generation electronic cigarette [19]. Aerosols were used to measure oxidative stress in human oral cells. The study found that e-cigarette aerosols produced formaldehyde, acetaldehyde, and acrolein, but did not contain detectable levels of free radicals. Only one of the eight liquids tested using a customized atomizer induced cytotoxicity against two human oral cells in-vitro.

Treatment of oral cells with the cytotoxic e-cigarette aerosol increased intracellular oxidative stress. Further, the effects depended on the age of the atomizer, where repeated use increased production of radical emissions and cytotoxicity. Overall, these results suggest that third-generation e-cigarettes may cause adverse effects in the oral cavity and normal e-cigarette use, which involves repeated use of the same atomizer to generate aerosol, may enhance the potential toxic effects of third-generation e-cigarettes. This study represents the challenges of in-vitro studies. Numerous factors may affect toxicity including the type of e-cigarette, the type of atomizer, the extent to which it is used, and the type of e-cigarette liquids.

\section{In-vivo studies}

Preclinical animal models have been used to study oral squamous cell cancer. These types of studies also offer the opportunity to study the effects of e-cigarette vapor. There are many kinds of oral cancer animal models [20], and establishing the ones that would be most informative for studying e-cigarettes represents an intriguing challenge.

Nevertheless, models could be used to establish shortterm exposure in naïve animals and those pre-exposed to cigarette smoke. The specific impact of vapor components could be studied including flavorants, free radicals and aldehydes present in e-cigarette vapor. Outcomes may include biomarkers of oxidative stress and oxidative damage, markers of inflammation, and oral cancer pathways. These models can reflect human exposure conditions including the absolute effect of e-cigarette exposure, dual use with tobacco smoke exposure, and post-initiation studies that might model the potential effects of e-cigarette on HNC for former cigarette smokers.

\section{Conclusion}

In this review, we suggest that the use of e-cigarettes may have a significant effect on the risk of head and neck cancer, one of the leading causes of cancer worldwide. Few studies have been conducted to measure these effects and while this represents a considerable gap in data that support or refute the use of e-cigarettes for harm reduction, it also demonstrates considerable opportunities for further research in both in-vitro, in-vivo and epidemiological sciences. 


\section{References}

1. Rettig EM, D'Souza G (2015) Epidemiology of head and neck cancer. Surg Oncol Clin N Am 24: 379-396.

2. Bray F, Ferlay J, Soerjomataram I, et al. (2018) Global cancer statistics 2018: GLOBOCAN estimates of incidence and mortality worldwide for 36 cancers in 185 countries. CA Cancer J Clin 68: 394-424.

3. Simard EP, Torre LA, Jemal A, et al. (2014) International trends in head and neck cancer incidence rates: Differences by country, sex and anatomic site. Oral Oncol 50: 387-403.

4. Cullen KA, Ambrose BK, Gentzke AS, et al. (2018) Notes from the field: Use of electronic cigarettes and any tobacco product among middle and high school students - United States, 20112018. MMWR Morb Mortal Wkly Rep 67: 1276-1277.

5. Villanti AC, Johnson AL, Ambrose BK, et al. (2017) Flavored tobacco product use in youth and adults: Findings from the first wave of the PATH study (2013-2014). Am J Prev Med 53: 139151.

6. Levy DT, Yuan Z, Li Y, et al. (2017) The prevalence and characteristics of e-cigarette users in the U.S. Int J Environ Res Public Health 14: 1200.

7. Ebersole J, Samburova V, Son Y, et al. (2020) Harmful chemicals emitted from electronic cigarettes and potential deleterious effects in the oral cavity. Tob Induc Dis 18: 41.

8. Schottenfeld DJF (2008) Cancer Epidemiology and Prevention. New York: Oxford University Press.

9. Sapkota A, Gajalakshmi V, Jetly DH, et al. (2007) Smokeless tobacco and increased risk of hypopharyngeal and laryngeal cancers: A multicentric case-control study from India. Int J Cancer 121: 1793-1798.

10. Bitzer ZT, Goel R, Reilly SM, et al. (2019) Emissions of free radicals, carbonyls, and nicotine from the NIDA standardized research electronic cigarette and comparison to similar commercial devices. Chem Res Toxicol 32: 130-138.
11. Pushalkar S, Paul B, Li Q, et al. (2020) Electronic cigarette aerosol modulates the oral microbiome and increases risk of infection. iScience 23: 100884.

12. Wadia R, Booth V, Yap HF, et al. (2016) A pilot study of the gingival response when smokers switch from smoking to vaping. $\mathrm{Br}$ Dent J 221: 722-726.

13. Bin Shabaib, M SSAL, Akram Z, et al. (2019) Clinical periodontal status and gingival crevicular fluid cytokine profile among cigarette-smokers, electronic-cigarette users and never-smokers. Arch Oral Boil 102: 212-217.

14. Lin SC, Liu CJ, Chiu CP, et al. (2004) Establishment of OC3 oral carcinoma cell line and identification of NF-kappa $b$ activation responses to areca nut extract. J Oral Pathol Med 33: 79-86.

15. Rouabhia M, Park HJ, Semlali A, et al. (2017) E-cigarette vapor induces an apoptotic response in human gingival epithelial cells through the caspase-3 pathway. J Cell Physiol 232: 1539-1547.

16. Tsai KYF, Hirschi Budge KM, Lepre AP, et al. (2020) Cell invasion, RAGE expression, and inflammation in oral squamous cell carcinoma (OSCC) cells exposed to e-cigarette flavoring. Clin Exp Dent Res.

17. Irani S, Barati I, Badiei M, et al. (2020) Periodontitis and oral cancer - current concepts of the etiopathogenesis. Oncol Rev 14: 465.

18. Michaud DS, Fu Z, Shi J, et al. (2017) Periodontal disease, tooth loss, and cancer risk. Epidemiol Rev 39: 49-58.

19. Urena JF, Ebersol LA, Silakov A, et al. (2020) Impact of atomizer age and flavor on in vitro toxicity of aerosols from a third-generation electronic cigarette against human oral cells. Chem Res Toxicol 33: 2527-2537.

20. Li Q, Dong H, Yang G, et al. (2020) Mouse tumor-bearing models as preclinical study platforms for oral squamous cell carcinoma. Front Oncol 10: 212.

DOI: $10.36959 / 915 / 574$

Copyright: (C) 2020 Muscat JE, et al. This is an open-access article distributed under the terms of the Creative Commons Attribution License, which permits unrestricted use, distribution, and reproduction in any medium, provided the original author and source are credited. 